\title{
Elevated rhythmic Ras activity in the suprachiasmatic nucleus of synRas transgenic mice: implications for the regulation of the mammalian circadian clock
}

\author{
Tsvetan Serchov*1, Antje Jilg², Jörg H Stehle ${ }^{2}$ and Rolf Heumann ${ }^{1}$
}

\author{
Address: ${ }^{1}$ Molecular Neurobiochemistry, Ruhr-University, Bochum, Germany and ${ }^{2}$ Institute of Anatomy III, J. W. Goethe-University, Frankfurt, \\ Germany \\ * Corresponding author
}

from Annual Meeting of the Study Group Neurochemistry. International Conference of the Gesellschaft für Biochemie und Molekularbiologie 2006 (GBM 2006): Molecular pathways in health and disease of the nervous system

Witten, Germany. 28-30 September 2006

Published: 23 March 2007

BMC Neuroscience 2007, 8(SuppI I):P34 doi:I0.I I86/I47I-2202-8-SI-P34

(C) 2007 Serchov et al; licensee BioMed Central Ltd.

Light is the main phase-adjusting stimulus of the circadian clock located in the suprachiasmatic nucleus (SCN). A candidate pathway transmitting photic information at the postsynaptic site in the SCN is the extracellular signalregulated kinase (ERK 1/2) which has been previously shown to be an essential element in the photoentrainment of the circadian rhythm. An upstream activator of the ERK signalling route is the small intracellular GTPase Ras. Here we observed that endogenous Ras activity in the SCN was subjected to rhythmic changes, reaching maximum levels at the late subjective day and minimum levels at the late subjective night (CT22). In order to investigate if Ras would modulate the circadian cycle, we used transgenic mice expressing constitutively activated Val-12 HaRas selectively in neurons (synRas mice). In these mice Ras activity was also cycling during the circadian rhythm yet, Ras activities were up-regulated at each time point measured. We investigated if this change in Ras activity translates into a behavioral phenotype by monitoring free-running activity rhythms under conditions of constant darkness. SynRas mice exhibited circadian rhythms in locomotor activities similar to WT mice. However, when challenged by applying a 15 minutes light pulse at CT22 to promote phase advance shifts, synRas mice were completely non-responsive. As a first step towards the possible intracellular mechanism of this behavioral change we analyzed ERK1/2 activities in more details: We found a 1,7-fold increase of circadian peak levels of ERK $1 / 2$ activities at CT10 and CT14 in synRas mice, while at minimum levels (CT18, CT22) no differences were found between ERK1/2 activities of WT and synRas mice. In WT animals the 15 minutes light pulse at CT22 resulted in rapid up regulations of Ras, ERK1/2 and CREB activities as described previously by others. However, in correlation with the lack of a behavioral response, ERK1/2 but not Ras and CREB activities remained unchanged in synRas mice, suggesting that Ras-dependent and Ras-independent pathways may co-exist to regulate ERK1/2 and behavioral phase shifts in response to the acute light treatment.

Next we investigated the length "tau" of the locomotor activity rhythm during constant darkness and found a slight shortening by about 10 minutes in synRas mice as compared to the WT littermates. Recently, "tau" has been discussed to be modulated by the interaction between glycogen synthase $3 \beta$ (GSK3 $\beta$ ) and a clock gene product (Per 2 ) that is involved in the determination of circadian phase durations. We describe here a down-regulation of GSK3 $\beta$ phosphorylation in synRas mice as a possible mechanism of "tau" shortening. Taken together, cycling of Ras activity at elevated levels in the SCN during the circadian rhythm results in a distinct pattern of behavioral phenotype changes correlating with de-regulated ERK1/2 or GSK3 $\beta$ activities. 